\title{
NOTE
}

\section{The Influence of Stern Layer Conductance on the Dielectrophoretic Behavior of Latex Nanospheres}

The influence of the Stern layer conductance on the dielectrophoretic behavior of sub-micrometer-sized latex spheres is examined. The dielectrophoretic response of the particles is measured and analyzed in terms of a model of surface conductance divided into discrete components related to the structure of the double layer. The effect of both co- and counterions in the bulk solution on the Stern layer conductance is demonstrated. $\odot 2002$ Elsevier Science (USA)

Key Words: dielectrophoresis; electrokinetics; conductance; electrical double layer.

A polarizable particle suspended in a nonuniform electric field experiences a force due to the interaction of the field and the induced dipole moment. The resulting movement of the particle is referred to as dielectrophoresis $(1,2)$. The dielectrophoretic force is positive (particles attracted to regions of high field strength) or negative (particles repelled) when the particle is more or less polarizable than the medium, respectively.

An example of dielectrophoresis is shown in Fig. 1, with fluorescent latex spheres suspended in $\mathrm{KCl}$ on a quadrupole electrode structure (3, 4). At low frequencies, the spheres are attracted to regions of high field at the edges of electrodes and between adjacent electrodes. At high frequencies the particles are repelled to the local low field region at the center of the electrode array (3).

This type of latex sphere, suspended in an electrolytic solution, undergoes a single interfacial relaxation process at a frequency related to the properties of particle and medium (2). The particles have a high surface charge density and therefore a polarizability greater than the aqueous suspending medium at low frequencies. As a result, the particles experience positive dielectrophoresis at low frequencies. At high frequencies the permittivity of the particle is significantly lower than that of water; the particles are therefore less polarizable and experience negative dielectrophoresis. The dielectrophoretic force changes sign at a characteristic frequency, generally referred to as the crossover frequency. Analysis of the crossover frequency as a function of medium conductivity can be used to characterize the polarizability and as a result the dielectric properties of a particle. This method of dielectrophoretic analysis has been demonstrated on sub-micrometer particles such as latex beads $(5,6)$ and viruses $(7-9)$.

Previous work $(5,10)$ has shown that the dielectrophoretic behavior of submicrometer latex spheres is dominated by the effects of charge movement across the particle surface. In this paper we demonstrate that conductance in the double layer is influenced not only by the movement of counterions at the particle surface but also by the net mobilities of both counterions and co-ions in the bulk medium.

The dielectrophoretic force $\mathbf{F}_{D E P}$ acting on a homogeneous, isotropic dielectric sphere is given by (2)

$$
\mathbf{F}_{D E P}=2 \pi r^{3} \varepsilon_{m} \operatorname{Re}\left[f_{C M}\right] \nabla|\mathbf{E}|^{2},
$$

where $r$ is the particle radius, $\varepsilon_{m}$ the permittivity of the suspending medium, $\mathbf{E}$ the local RMS electric field, and $\operatorname{Re}\left[f_{C M}\right]$ the real part of the Clausius-Mossotti factor, given by

$$
f_{C M}=\frac{\varepsilon_{p}^{*}-\varepsilon_{m}^{*}}{\varepsilon_{p}^{*}+2 \varepsilon_{m}^{*}},
$$

where $\varepsilon_{m}^{*}$ and $\varepsilon_{p}^{*}$ are the complex permittivities of the medium and particle respectively and a general complex permittivity is given by $\varepsilon^{*}=\varepsilon-i \sigma / \omega$, with $\omega$ the angular frequency of the applied field, $\sigma$ the conductivity, and $\varepsilon$ the permittivity. This factor describes the frequency dependence of the dipole moment of the particle and the resulting dielectrophoretic behavior of the particle.

Experimental measurement of the dielectrophoresis of colloidal latex particles has demonstrated that for very small particles (less than $500 \mathrm{~nm}$ in diameter) the effective particle conductivity varies as a function of the medium conductivity $(5,10)$. Recent work by Hughes et al. (10) has demonstrated that the effective conductivity of a latex particle can be described as the sum of three components: the bulk conductivity of the particle, $\sigma_{\text {pbulk }}$, and surface components due to the movement of charge in the diffuse double layer (conductance $K_{s}^{d}$ ) and the Stern layer (conductance $K_{s}^{i}$ ). The total conductivity of the particle is then written as

$$
\sigma_{p}=\sigma_{p b u l k}+\frac{2 K_{s}^{i}}{r}+\frac{2 K_{s}^{d}}{r}
$$

For latex spheres, the bulk conductivity is negligible and the effective conductivity of the particle is dominated by the surface conductance. The conductance of the diffuse layer can be expressed as (11)

$$
K_{s}^{d}=\frac{\left(4 F^{2} c z^{2} D^{d}\left(1+3 m / z^{2}\right)\right)}{R T \kappa}\left(\cosh \left[\frac{z F \zeta}{2 R T}\right]-1\right),
$$

where $D^{d}$ is the ion diffusion coefficient, $z$ the valency of the counterion, $F$ the Faraday constant, $R$ the molar gas constant, and $T$ the temperature. $\kappa$ is the reciprocal Debye length, given by

$$
\kappa=\sqrt{\left(\frac{2 c z F^{2}}{\varepsilon R T}\right)}
$$

$c$ is the electrolyte concentration $\left(\mathrm{mol} \mathrm{m}^{-3}\right)$, and $\zeta$ is the electrostatic potential at the boundary of the slip plane. The dimensionless parameter $m$ describes the electro-osmotic contribution to $K_{s}^{d}$ and is given by (11)

$$
m=\left(\frac{R T}{F}\right)^{2} \frac{2 \varepsilon_{m}}{3 \eta D^{d}}
$$

where $\eta$ is the viscosity. In previous work (10) the conductance of the Stern layer was given by the expression

$$
K_{s}^{i}=u \mu_{s}^{i}
$$



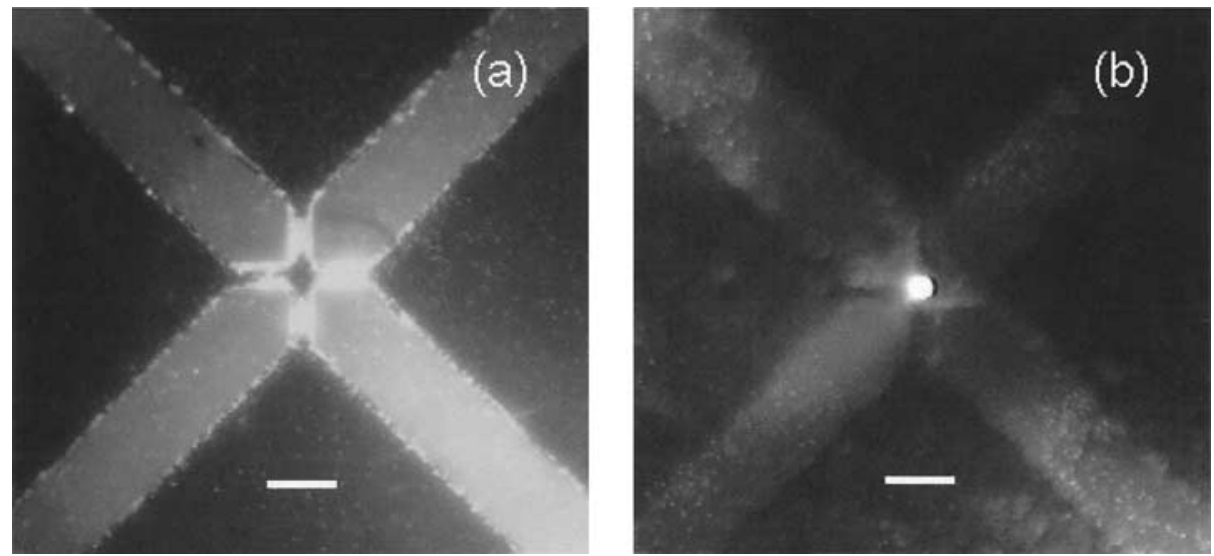

FIG. 1. Fluorescence micrographs showing positive and negative dielectrophoresis of 216-nm-diameter latex spheres. Four gold electrodes (visible as four bar-shaped, paler regions terminating in points) are energized such that opposing electrodes share the same phase, while adjacent electrodes are in antiphase. Figure 1a shows positive dielectrophoresis (particles attracted to regions of high electric field, that is, in the regions between adjacent electrodes), which occurs at lower frequencies; Fig. 1b shows negative dielectrophoresis (repulsion from regions of high electric field, causing collection at a field null at the center of the electrodes) which occurs at higher frequencies. The transition frequency between these modes of behavior is called the crossover frequency, and is related to the dielectric properties of particle and medium. The beads were suspended in a $1 \mathrm{mS} \mathrm{m}^{-1} \mathrm{KCl}$ solution, at frequencies of 1 and $10 \mathrm{MHz}$ and applied potential 5 $\mathrm{V}_{\mathrm{pk}-\mathrm{pk}}$, respectively. Scale bar: $20 \mu \mathrm{m}$.

where $u$ is the surface charge density and $\mu_{s}^{i}$ is the mobility of the counterion. However, analysis of experimental results using latex spheres suspended in different electrolytes with identical counterions but different co-ions (5) shows that Eq. [6] does not describe these experimental results correctly.

Returning to the definition of the Stern layer conductance, the Dukhin number $D u$ (11) for a Stern layer containing one ion species $i$ is given by

$$
D u=\frac{u \mu_{s}^{i}}{2 r z^{i} F c^{i} \mu_{m}^{i}},
$$

where $\mu_{s}^{i}$ and $\mu_{m}^{i}$ are the mobilities of the ion species in the Stern layer and bulk medium, respectively. The Dukhin number describes the ratio between surface and bulk conductances and is given by (11)

$$
D u=\frac{K_{s}^{i}}{r \sigma_{m}},
$$

where $\sigma_{m}$ is the conductivity of the bulk electrolyte. Combining Eqs. [7] and [8] and rearranging, we obtain the following expression for $K_{s}^{i}$ :

$$
K_{s}^{i}=\frac{u \mu_{s}^{i} \sigma_{m}}{2 z^{i} F c^{i} \mu_{m}^{i}} .
$$

If the electrolyte is symmetrical, it is possible to replace the conductivity term and concentration $c^{i}$ with molar conductivity $\ddot{\mathrm{E}}\left(\mathrm{S} \mathrm{m}^{2} \mathrm{~mol}^{-1}\right)$ :

$$
K_{s}^{i}=\frac{u \mu_{s}^{i} \Lambda}{2 z^{i} F \mu_{m}^{i}} .
$$

Values of $\Lambda$ are constant for given electrolytes (a table of values is given by Bockris and Reddy (12)). Equation [10] reduces to [6] only if the values of the mobilities of the co- and counterions are equal, in which case the value of

$$
\frac{\Lambda}{2 z^{i} F \mu_{m}^{i}}
$$

goes to 1 .

The validity of Eq. [10] was tested by measuring the dielectrophoretic crossover frequency for latex spheres in different electrolytes. The latex spheres (Molecular Probes, Oregon) were $216 \mathrm{~nm}$ in diameter and loaded with fluorescent dye. The suspending electrolytes were aqueous solutions of $\mathrm{KCl}$ and
$\mathrm{K}_{2} \mathrm{HPO}_{4}-\mathrm{KH}_{2} \mathrm{PO}_{4}$ (a $50: 50$ mixture of monobasic and dibasic potassium phosphate) with conductivities in the range $10^{-4}$ to $10^{-2} \mathrm{~S} \mathrm{~m}^{-1}$. The $\mathrm{pH}$ of the two solutions was measured at 6.5 and 7.1 for the $\mathrm{KCl}$ and $\mathrm{K}_{2} \mathrm{HPO}_{4}-\mathrm{KH}_{2} \mathrm{PO}_{4}$, respectively; since the $\mathrm{pH}$ of the media are similar, we assume that the number of dissociated carboxyl groups on the beads is similar for beads in both media. The suspensions of particles were placed on microfabricated electrode structures energized using a signal generator (3-5). The particle movement was observed using a Photonic Science Coolview HS light-intensifying camera and a fluorescence microscope.

The crossover frequencies of the beads in the two media are shown in Fig. 2. Three key features are used in the best-fit analysis of undescribed homogeneous spheres: the value of the crossover frequency in low-conductivity media, the gradient of the rise in crossover as a function of increasing medium conductivity, and the conductivity at which the crossover drops by a decade or more. However, where the internal properties of the particle are known and we are interested in determining the surface properties only, we are interested only in the crossover behaviour below the characteristic drop in conductivity. In this region, the dielectrophoretic behavior is dictated by the surface charge density which determines the low-conductivity crossover, (or " $y$ intercept" in Fig. 2) and the $\zeta$ potential affects the apparent increase in particle conductivity with medium conductivity (the "slope" of the graph in Fig. 2). The conductivity at which the frequency drops is a factor of the two surface conductance components and provides no extra information; where the internal properties of the particle are known, we need only use two parameters - the $y$ intercept and slope-to determine the surface conductances by best-fit methods.

Best-fit lines of Eq. [1], using Eqs. [3] and [4] to describe the particle conductivity, are also shown. Relative permittivities for the suspending electrolyte and particle, of 78 and 2.55, respectively, were assumed. The best values were $K_{s}^{i}=0.85( \pm 0.15) \mathrm{nS}$ and $\zeta=110( \pm 5) \mathrm{mV}$ for $\mathrm{KCl}$ and $K_{s}^{i}=1.25( \pm 0.1) \mathrm{nS}$ and $\zeta=120( \pm 10) \mathrm{mV}$ for $\mathrm{K}_{2} \mathrm{HPO}_{4}-\mathrm{KH}_{2} \mathrm{PO}_{4}$. We can analyze these values to determine the validity of Eq. [10]. Since carboxylated beads are negatively charged, the ion species involved in surface conduction is $\mathrm{K}^{+}$. We assume in modeling the data that the parameters for $\zeta$ potential and $K_{s}^{i}$ are constant over the range of conductivities studied; in reality, both will reduce slightly as the conductivity is increased, but the magnitude of this change over the range of molarities of the electrolytes used should be relatively small (see (11) section 4.6e). For latex spheres suspended in $\mathrm{KCl}$ and $\mathrm{K}_{2} \mathrm{HPO}_{4}-\mathrm{KH}_{2} \mathrm{PO}_{4}$, Eq. [6] indicates that the Stern layer conductance is the same and that the influence of this conductance on the dielectrophoretic behavior should also be the same. However, 


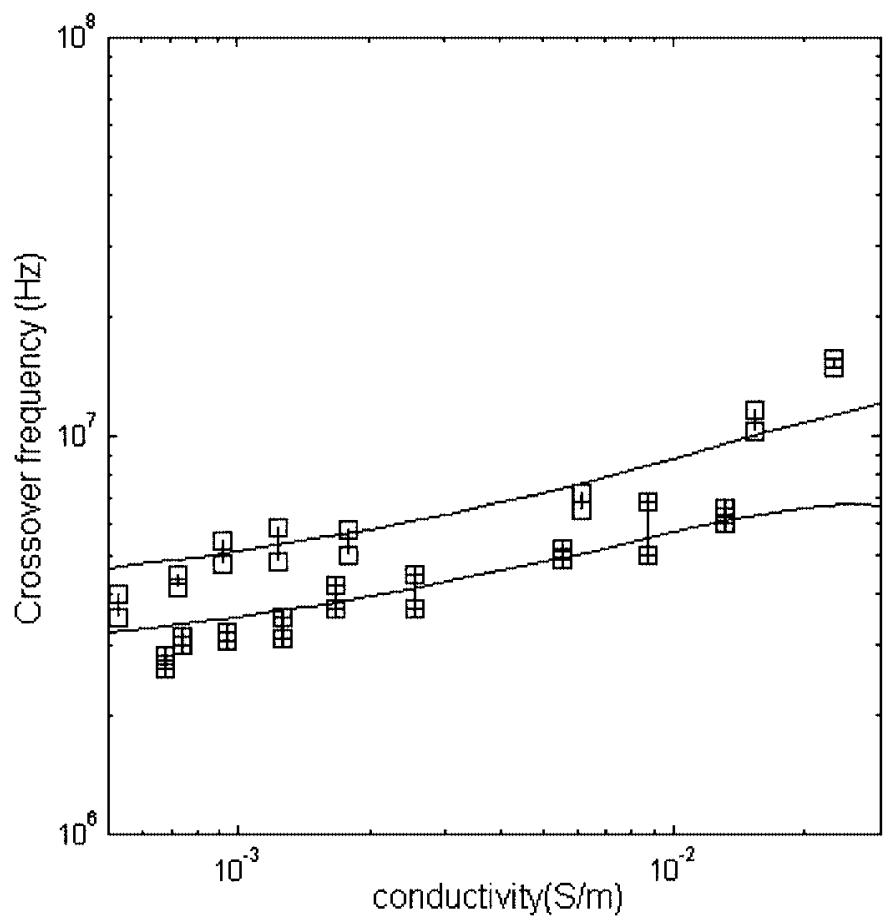

FIG. 2. Experimental DEP crossover spectra for 216-nm-diameter latex beads. The vertical lines show the range of frequencies in which dielectrophoretic crossover was observed for beads suspended in $\mathrm{KCl}$ (filled squares) and $\mathrm{K}_{2} \mathrm{HPO}_{4}-\mathrm{KH}_{2} \mathrm{PO}_{4}$ (empty squares) solutions of varying conductivity. The diagonal curves indicate the best-fit model to these data. The values for best fit indicate that the values of $K_{s}^{i}$ and $\zeta$ are $0.85 \mathrm{nS}$ and $100 \mathrm{mV}$ for the beads suspended in $\mathrm{KCl}$ and $1.25 \mathrm{nS}$ and $120 \mathrm{mV}$ in $\mathrm{K}_{2} \mathrm{HPO}_{4}-\mathrm{KH}_{2} \mathrm{PO}_{4}$, respectively. In both cases the bead permittivity was $2.25 \varepsilon_{0}$ and the internal conductivity was negligible.

the best-fit values from the experimental data give the ratio between $K_{s}^{i}$ in $\mathrm{K}_{2} \mathrm{HPO}_{4}-\mathrm{KH}_{2} \mathrm{PO}_{4}$ and $\mathrm{KCl}$ as 1.47 .

If we now consider the definition of the Stern layer conductance given by Eq. [10], we would expect that the ratio between $K_{s}^{i}$ in $\mathrm{K}_{2} \mathrm{HPO}_{4}-\mathrm{KH}_{2} \mathrm{PO}_{4}$ and $\mathrm{KCl}$ to be given by the ratio of the molar conductivities of the two electrolytes, all other factors being equal. The molar conductivities of $\mathrm{K}_{2} \mathrm{HPO}_{4}-\mathrm{KH}_{2} \mathrm{PO}_{4}$ and $\mathrm{KCl}$ are 21.2 and $14 \mathrm{mS} \mathrm{m} \mathrm{mol}^{-1}$, respectively (12), giving an expected ratio between the Stern layer conductances of 1.51 in good agreement with the best fit values.

To ensure that the effect being observed is not merely the result of the variation in ion concentration for the two electrolytes, the crossover data were analyzed as a function of $\mathrm{K}^{+}$concentration rather than conductivity; this graph (not shown) demonstrates trends similar to the frequency-conductivity plot shown in Fig. 2, indicating that the observed effects are not due to the difference in $\mathrm{K}^{+}$concentrations in the two electrolytes.

Application of the same analysis to the data presented in Green and Morgan (5) for similar latex beads in solutions of $\mathrm{KCl}, \mathrm{NaCl}$, and $\mathrm{K}_{2} \mathrm{HPO}_{4}-\mathrm{KH}_{2} \mathrm{PO}_{4}$ (not shown) indicates similar results to those presented here. These additional data indicate the same relationship between $\mathrm{KCl}$ and $\mathrm{K}_{2} \mathrm{HPO}_{4}-\mathrm{KH}_{2} \mathrm{PO}_{4}$ as described above; i.e., matching gives different values for $K_{s}^{i}$, for the same counterion but different co-ions. In addition, the data also indicate that $\mathrm{NaCl}$ also can be fitted using the arguments presented in this paper, i.e., that the values of $K_{s}^{i}$ for $\mathrm{NaCl}$ and $\mathrm{KCl}$ are similar, for the same counterion but different co-ions.

Measurement of the electrophoretic mobility of latex spheres of various sizes (5) demonstrated that the surface charge density of latex spheres is in the range 22-40 mC m$~_{-2}$, with $33 \mathrm{mC} \mathrm{m}^{-2}$ falling within the tolerance range of all bead sizes. Considering the above experimental data for beads in $\mathrm{KCl}$ and the deduced values of $K_{s}^{i}$, it is possible to estimate the value of the Stern layer mobility. Consider the data for latex beads in $\mathrm{KCl}$ presented above. $\mathrm{KCl}$ has a molar conductivity of $14.1 \mathrm{mS} \mathrm{m} \mathrm{mol}^{-1}$ and the bulk mobility of the $\mathrm{K}^{+}$ counterion is $7.69 \times 10^{-8} \mathrm{~m}^{2} \mathrm{~V}^{-1} \mathrm{~s}^{-1}$. Using these values with the estimated value for $K_{s}^{i}$, we calculate from Eq. [9] a value for ion mobility within the Stern layer of $2.73 \times 10^{-8} \mathrm{~m}^{2} \mathrm{~V}^{-1} \mathrm{~s}^{-1}$ or approximately $35 \%$ of the bulk ion mobility. This result is in keeping with Lyklema's assertion (11) that the mobility of counterions in the Stern layer should be "close to, or somewhat lower than, the corresponding bulk values". If we calculate the value of $K_{s}^{i}=u \mu_{s}^{i}$ using the above data, we find $\mu_{s}^{i}$ of $2.575 \times 10^{-8} \mathrm{~m}^{2} \mathrm{~V}^{-1} \mathrm{~s}^{-1}$; i.e., the value of

$$
\frac{\Lambda}{2 z^{i} F \mu_{m}^{i}}
$$

is approximately 0.95 and the approximation in Eq. [6] holds. This result is important in that it demonstrates the mechanism by which the co-ion in solution affects the Stern layer mobility, and hence the surface conductance, in line with observed results.

\section{ACKNOWLEDGMENTS}

This work was funded by a Royal Society research grant to M.P.H. and a Royal Academy of Engineering Postdoctoral Research fellowship to N.G.G. The authors thank David Lamb for the use of the fluorescence microscope.

\section{REFERENCES}

1. Pohl, H. A., "Dielectrophoresis." Cambridge Univ. Press, Cambridge, 1978.

2. Jones, T. B., "Electromechanics of particles." Cambridge Univ. Press, Cambridge, 1995.

3. Green, N. G., Ramos, A., and Morgan, H., J. Phys. D: Appl. Phys. 33, 632-641 (2000).

4. Hughes, M. P., and Morgan, H., J. Phys. D: Appl. Phys. 31, 2205-2210 (1998).

5. Green, N. G., and Morgan, H., J. Phys. Chem. 103, 41-50 (1999).

6. Hughes, M. P., and Morgan, H., Anal. Chem. 71, 3441-3445 (1999).

7. Morgan, H., and Green, N. G., J. Electros. 42, 279-293 (1997).

8. Hughes, M. P., Morgan, H., Rixon, F. J., Burt, J. P. H., and Pethig, R., Biochim. Biophys. Acta 1425, 119-126 (1998).

9. Hughes, M. P., Morgan, H., and Rixon, F. J., Eur. Biophys. J. 30, 268-272 (1998).

10. Hughes, M. P., Morgan, H., and Flynn, M. F., J. Colloid Interface Sci. 220, 454-457 (1999).

11. Lyklema, J., "Fundamentals of Interface and Colloid Science." Academic Press, London, 1995.

12. Bockris, J. O'M., and Reddy, A. K. N., "Modern Electrochemistry." Plenum, New York, 1970.

Michael Pycraft Hughes*,1 Nicolas Gavin Green $\dagger$

*Centre for Biomedical Engineering,

University of Surrey, Guildford

GU2 7 X H, United Kingdom

$\dagger$ Bioelectronics Research Centre,

Department of Electronics and Electrical

Engineering, University of Glasgow,

Glasgow G12 8LT, United Kingdom

Received August 24, 2001; accepted February 27, 2002;

published online April 29, 2002

\footnotetext{
${ }^{1}$ To whom correspondence should be addressed. Fax: +44 1483689395 . E-mail: m.hughes@surrey.ac.uk.
} 